\title{
La economía de Canadá
}

$\mathrm{T}$ anto por el desarrollo de su economía como por el nivel de vida de sus habitantes, Canadá es la séptima economía del mundo y miembro del Club de las Siete Naciones Industrializadas, el G-7. Por su tamaño, es el segundo país más grande del orbe después de Rusia: con una extensión territorial cinco veces mayor a la de México y similar a la de Estados Unidos, país con el que comparte cerca de 9000 kilómetros de frontera. Canadá posee así una posición geográfica privilegiada, rodeado por los océanos Atlántico, Pacífico y Ártico, mientras la ruta marítima del San Lorenzo penetra, en el este, más de 3000 kilómetros de su frontera sur. Vía la ruta polar, Canadá tiene una ubicación estratégica entre Rusia y Estados Unidos.

Canadá posee una amplia dotación de recursos naturales y minerales, base sobre la cual el país construyó su desarrollo y cuyo acceso, no obstante lo vasto de su territorio, ha estado garantizado por una extensa red de vías de comunicación. Canadá es rico en recursos forestales, en gas natural y petróleo y en metales y minerales. La infraestructura física canadiense está considerada como la mejor del G-7.

El país cuenta con dos sistemas transcontinentales de ferrocarriles que cubren 73000 kilómetros: los Ferrocarriles Nacionales Canadienses y la Compañía Canadiense del Pacífico. Este transporte moviliza alrededor de 270 millones de toneladas de carga anualmente; mientras que 10 millones de camiones que cruzan la frontera con Estados Unidos al año, transportan 400 millones de toneladas equivalente a 70 por ciento del valor del comercio. El país tiene un millón de kilómetros en carreteras, 35 por ciento de los cuales están pavimentados. Canadá cuenta además con 25 puertos profundos y 650 más pequeños. En

* Investigadora del Departamento de Estudios del Pacífico de la Universidad de Guadalajara.

ORCID http://orcid.org/0000-0003-4926-0594 telecomunicaciones, Canadá posee un excelente servicio telefónico.

En comparación con Estados Unidos y México, la población de Canadá es la más pequeña de los tres países: 30 millones de habitantes que contrastan con los 267 millones de estadounidenses y los 94 millones de mexicanos. Así, de los tres, es la nación que posee la menor relación tierra/hombre: tres habitantes por kilómetro cuadrado (cuadro 1). Sin embargo, más de 90 por ciento de la población canadiense está localizada a 160 kilómetros de la frontera de su vecino del sur. La fuerza de trabajo canadiense está constituida por 14 millones de personas, mientras en Estados Unidos y México ésta es de 129 y 36 millones, respectivamente. La mano de obra de Canadá tiene un alto nivel de educación; la población mayor de quince años posee al menos nueve años de educación. ${ }^{1}$

De acuerdo con la estructura productiva del país, únicamente 4 por ciento de la mano de obra canadiense está empleada en el sector agrícola, en tanto el sector industrial absorbe 24 por ciento y el 73 por ciento restante se emplea en el sector servicios. Ésta es una estructura de empleo muy similar a la de Estados Unidos. En contraste, México todavía emplea 22 por ciento de su fuerza de trabajo en la agricultura, mientras los servicios absorben únicamente 54 por ciento (cuadro 2 ).

A partir de la Segunda Guerra Mundial y sobre una amplia base de recursos naturales, una población educada y una infraestructura física de elevada calidad, Canadá alcanzó un crecimiento económico impresionante tanto en la manufactura como en los servicios, transformándose de una economía rural a una industrial y urbana. La economía canadiense destaca en la producción aeroespacial, la industria de máquinas, la industria electrónica y la de telecomunicaciones. En las industrias 
Cuadro 1

Indicadores básicos

América del Norte

1997

\begin{tabular}{|c|c|c|c|c|}
\hline Variable & Unidad & $\begin{array}{l}\text { Estados } \\
\text { Unidos }\end{array}$ & Canadá & México \\
\hline 1. Territorio & Miles $\mathrm{Km}^{2}$ & $9,159.1$ & $9,221.0$ & $1,908.7$ \\
\hline Forestal & Porcentaje (\%) & 23.0 & 27.0 & 29.0 \\
\hline Promedio anual de deforestación (1990-1995) & Porcentaje (\%) & -0.3 & -0.1 & 0.9 \\
\hline 2. Población & Miles & 267.636 .0 & 30.287 .0 & 94.349 .0 \\
\hline Habitantes por $\mathrm{Km}^{2}$ & Personas & 28.0 & 3.0 & 47.0 \\
\hline Crecimiento (1990-1997) & Porcentaje (\%) & 1.0 & 1.2 & 1.8 \\
\hline Esperanza de vida al nacer & Años & 76.0 & 79.0 & 72.0 \\
\hline Tasa mortalidad infantil 1000 nacidos vivos & & 7.0 & 6.0 & 31.0 \\
\hline Inscripción de las niñas en primaria & Porcentaje (\%) & 95.0 & 94.0 & nd \\
\hline Mujer en la fuerza de trabajo & Porcentaje (\%) & 46.0 & 45.0 & 32.0 \\
\hline 3. Economía & & & & \\
\hline Producto Nacional Bruto (PNB) & Millones de dólares & $7,783,092.0$ & $594,976.0$ & $348,627.0$ \\
\hline PNB per cápita & Dólares & $29,080.0$ & $19,640.0$ & $3,700.0$ \\
\hline PNB per cápita & Dólares internacionales & $29,080.0$ & $21,750.0$ & $8,110.0$ \\
\hline Crecimiento promedio anual (1990-1997) & Porcentaje (\%) & 1.7 & 0.8 & 0.2 \\
\hline Agricultura en la economía & Porcentaje del PIB (\%) & 2.0 & 3.0 & 5.0 \\
\hline Empleo: fuerza de trabajo & Miles & $129,588.0$ & $13,941.0$ & $35,904.0$ \\
\hline En la agricultura & Porcentaje (\%) & 2.7 & 3.8 & 23.2 \\
\hline En la industria & Porcentaje (\%) & 23.9 & 23.2 & 22.7 \\
\hline En servicios & Porcentaje (\%) & 73.4 & 73.0 & 54.1 \\
\hline Inversión en la economía & Porcentaje del PIB (\%) & 18.0 & 18.0 & 26.0 \\
\hline Inversión del sector privado en el total & Porcentaje (\%) & 85.9 & & \\
\hline Tasa de ahorro bruta & Porcentaje (\%) & 17 & 18.4 & 23.6 \\
\hline Inflación promedio anual 1990-1997 & Porcentaje (\%) & 2.1 & 1.4 & 19.3 \\
\hline Gastos militares como porcentaje del PNB, 1995 & Porcentaje (\%) & 3.8 & 1.7 & 1.0 \\
\hline 4. Sector externo & & & & \\
\hline Exportaciones & Millones de dólares & $680,000.0$ & $214,400.0$ & $110,431.0$ \\
\hline Exportaciones manufacturares como \% del total & Porcentaje (\%) & 81.0 & 63.0 & 81.0 \\
\hline Crecimiento $1992-1997$ & Porcentaje (\%) & 8.0 & 8.1 & 17.8 \\
\hline Importaciones & Millones de dólares & $877,000.0$ & $201,000.0$ & $109,808.0$ \\
\hline Crecimiento $1992-1997$ & Porcentaje (\%) & 9.4 & 8.8 & 13.9 \\
\hline Comercio de bienes y servicios & & & & \\
\hline (Exportaciones+Importaciones) como \% del PIB medido en PPP & Porcentaje (\%) & 20.4 & 62.9 & 29.3 \\
\hline Balanza en cuenta corriente $\%$ del PIB & Porcentaje (\%) & -2.0 & -1.5 & -1.8 \\
\hline Inversión extranjera directa como \% del PIB & Porcentaje (\%) & 1.2 & 1.2 & 3.1 \\
\hline Tipo de cambio promedio & Moneda local por dólar & - & 1.3 & 7.9 \\
\hline Promedio 1990-1996 & Moneda local por dólar & - & 1.3 & 4.2 \\
\hline Ingresos por turismo & Millones de dólares & $75,056.0$ & $8,928.0$ & $7,593.0$ \\
\hline Visitantes & Miles & $48,409.0$ & $17,610.0$ & $19,381.0$ \\
\hline 5. Otros indicadores de nivel de vida & & & & \\
\hline Consumo de electricidad per cápita, 1996 & kwh & $11,796.0$ & $15,129.0$ & $1,381.0$ \\
\hline Líneas telefónicas & Por 1000 personas & 644.0 & 609.0 & 96.0 \\
\hline Automóviles (1995) & Por 1000 personas & 513.0 & 466.0 & 94.0 \\
\hline Televisiones (1994) & Por 1000 personas & 817.0 & 685.0 & 163.0 \\
\hline Computadoras personales & Por 1000 personas & 407.0 & 270.6 & 37.3 \\
\hline Médicos (1996) & Por 1000 personas & 2.6 & 2.1 & 1.6 \\
\hline Carreteras pavimentadas como $\%$ del total & Porcentaje del total (\%) & 60.5 & 35.3 & 37.4 \\
\hline
\end{tabular}

$\mathrm{PPP}=$ Paridad del poder de compra; $\mathrm{PIB}=$ Producto Interno Bruto; $\mathrm{PNB}=$ Producto Nacional Bruto

Fuentes: World Bank, World Bank Atlas 1999; OECD Economic Surveys, Canadá, 1999.

Melba, Falck y Agustina, Rodríguez, "México en la economía internacional.

Una visión estadística". Departamento de Estudios del Pacífico, Cucsh , UdeG, en prensa. 


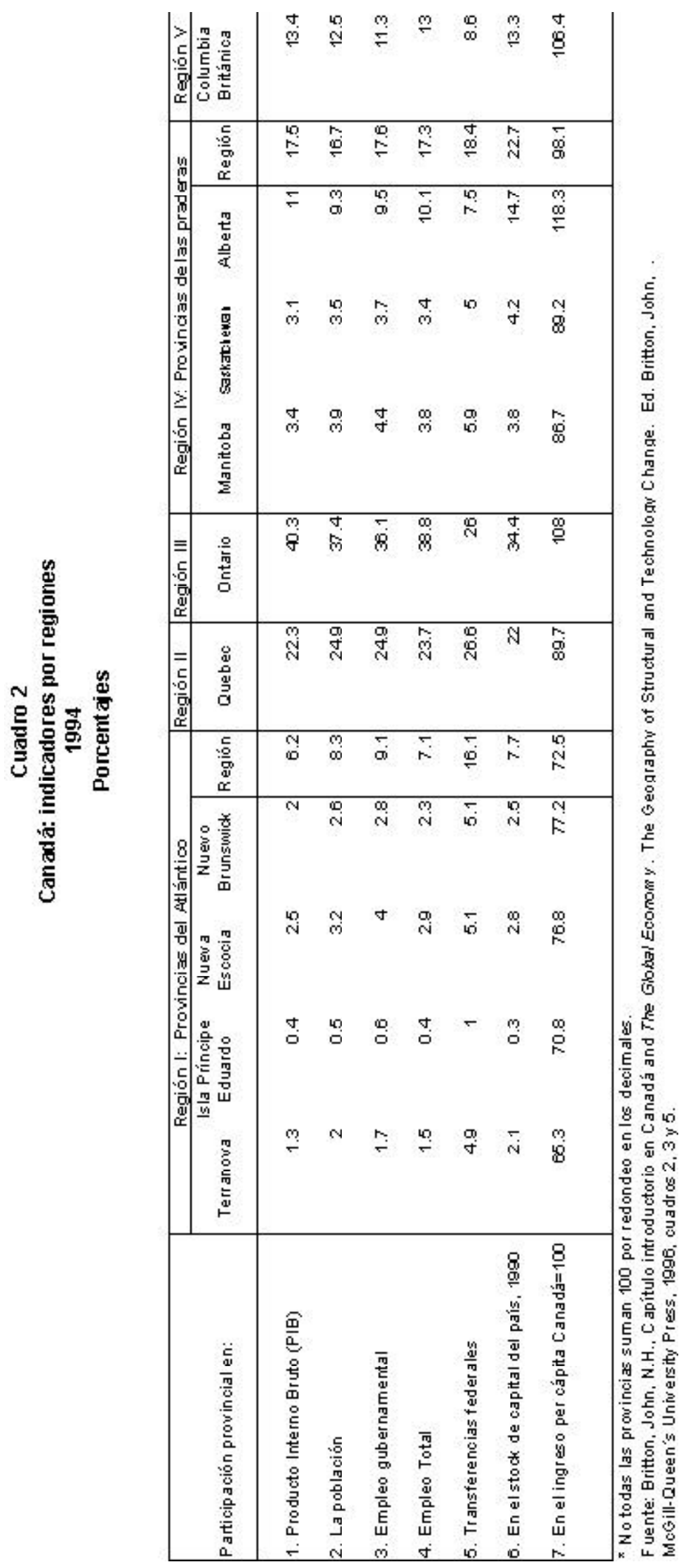


basadas en los recursos naturales, sobresalen la del gas y la del petróleo, la alimenticia y la forestal.

En la década de los sesenta la economía canadiense creció a una tasa promedio de 5.6 por ciento, la cual disminuyó a 4.7 por ciento en la siguiente década por el impacto de la crisis del petróleo. No obstante, la crisis del petróleo de 1979 y la recesión mundial de principios de los ochenta tuvieron un impacto mayor sobre la economía de Canadá, que por primera vez desde los sesenta experimentó una caída del PIB de alrededor de 3 por ciento (gráfica 1 ). Así, el crecimiento promedio en la década de los ochenta se situó en 3.1 por ciento. Sin embargo, es en la década de los noventa cuando Canadá experimenta menores tasas de crecimiento, en promedio 2.3 por ciento, mientras su vecino del sur gozaba de la más larga expansión económica.

Este crecimiento, aún considerando su tendencia a la baja, se ha traducido en un elevado estándar de vida para la población canadiense, representado por un nivel de ingreso per cápita en dólares internacionales (que miden el poder adquisitivo) de 21750 , que constituye 75 por ciento del de los estadounidenses y es 2.7 veces superior al de los mexicanos (cuadro 1). Así, de los nueve millones de familias canadienses, 98 por ciento poseen televisión y están conectadas al sistema de televisión por cable, mientras que alrededor de 60 por ciento de las familias tienen una computadora personal.

Por otra parte, todos los canadienses tienen acceso al sistema de seguro de salud Medicare, el cual es financiado por los impuestos sobre la renta tanto federales como provinciales, aunque cada gobierno provincial es responsable del manejo y la provisión de los servicios médicos.

El crecimiento económico de Canadá ha estado basado en la orientación hacia el exterior y en el financiamiento a través de la inversión extranjera. Para 1997, de los tres países de América del Norte, Canadá es el que presenta un mayor grado de apertura. Tomando como parámetro el flujo de comercio, las exportaciones más las importaciones como porcentaje del Producto Interno Bruto (PIB), éste representa para Canadá un 63 por ciento; México sigue en importancia con 29 por ciento y Estados Unidos es la economía más cerrada de los tres, con un grado de apertura de 20 por ciento. No obstante este último por el tamaño de su economía, trece veces superior a la canadiense, es el principal exportador e importador del mundo. En 1997, mientras Estados Unidos exportaba 680000 millones de dólares, las exportaciones canadienses ascendían a 214000 millones de dólares y las mexicanas a 110 000. Por el lado de las compras al exterior, en el mismo año el nivel correspondiente a Estados Unidos fue de 877000 millones de dólares, mientras que Canadá y México presentan valores de 201 y 110000 millones de dólares, respectivamente (cuadro 1).

Dos características destacan en el comercio exterior de Canadá. Aunque dos tercios de sus exportaciones son manufactureras, todavía éstas son altamente dependientes de los recursos primarios y no poseen un alto valor agregado. La mayor dependencia de Canadá de las exportaciones basadas en recursos naturales lo hacen más vulnerable a las variaciones en los 
precios. Además, existe una fuerte concentración de los flujos de comercio exterior en un solo mercado: Estados Unidos; situación similar a la que enfrenta México, aunque la intensidad de la relación comercial de Canadá y Estados Unidos es mucho mayor que la de este último con México. El flujo de comercio de Estados Unidos con Canadá es mayor al de Estados Unidos con todos los países de la Unión Europea. La intensidad de la relación comercial y de inversión entre los dos países se vio incrementada por la firma del Tratado de Libre Comercio (TLC) entre ambos en 1989 y del Tratado de Libre Comercio de América del Norte (TLCAN) en 1994.

El financiamiento del crecimiento económico de Canadá ha estado basado en la inversión extranjera. Entre las diez empresas más importantes de Canadá, cuatro son extranjeras y se estima que los inversionistas externos controlan cerca de una cuarta parte de los activos no financieros de Canadá. Para 1998 se calcula que el stock de la inversión extranjera directa fue de 142000 millones de dólares o una cuarta parte del PIB. A partir de la firma del TLC, Estados Unidos y Canadá adoptaron el principio de trato nacional a la inversión extranjera y con el TLCAN se expandieron las áreas de inversión y los derechos de los inversionistas, aunque se ha mantenido siempre el control por parte de Canadá de la industria cultural, además de que el régimen regulatorio a la inversión extranjera afecta también a los sectores de telecomunicaciones, aviación, minería, pesca y radiodifusión.

\section{Canadá regional ${ }^{2}$}

Una de las principales características de la economía canadiense es su diversidad regional. Entre las diez provincias y los tres territorios que constituyen Canadá, pueden distinguirse cinco regiones, clasificadas por la dotación de recursos y la especialización en la producción: a) las provincias de la región del Atlántico: Nueva Escocia, Nuevo Brunswick, Terranova, Labrador y la isla del príncipe Eduardo; b) la Provincia de Quebec; c) la Provincia de Ontario; d) las provincias de las Praderas: Manitoba, Saskatchewan, Alberta y los territorios del noroeste, y e) la provincia de Columbia Británica y el territorio del Yukon (Ver mapa y cuadro 2).

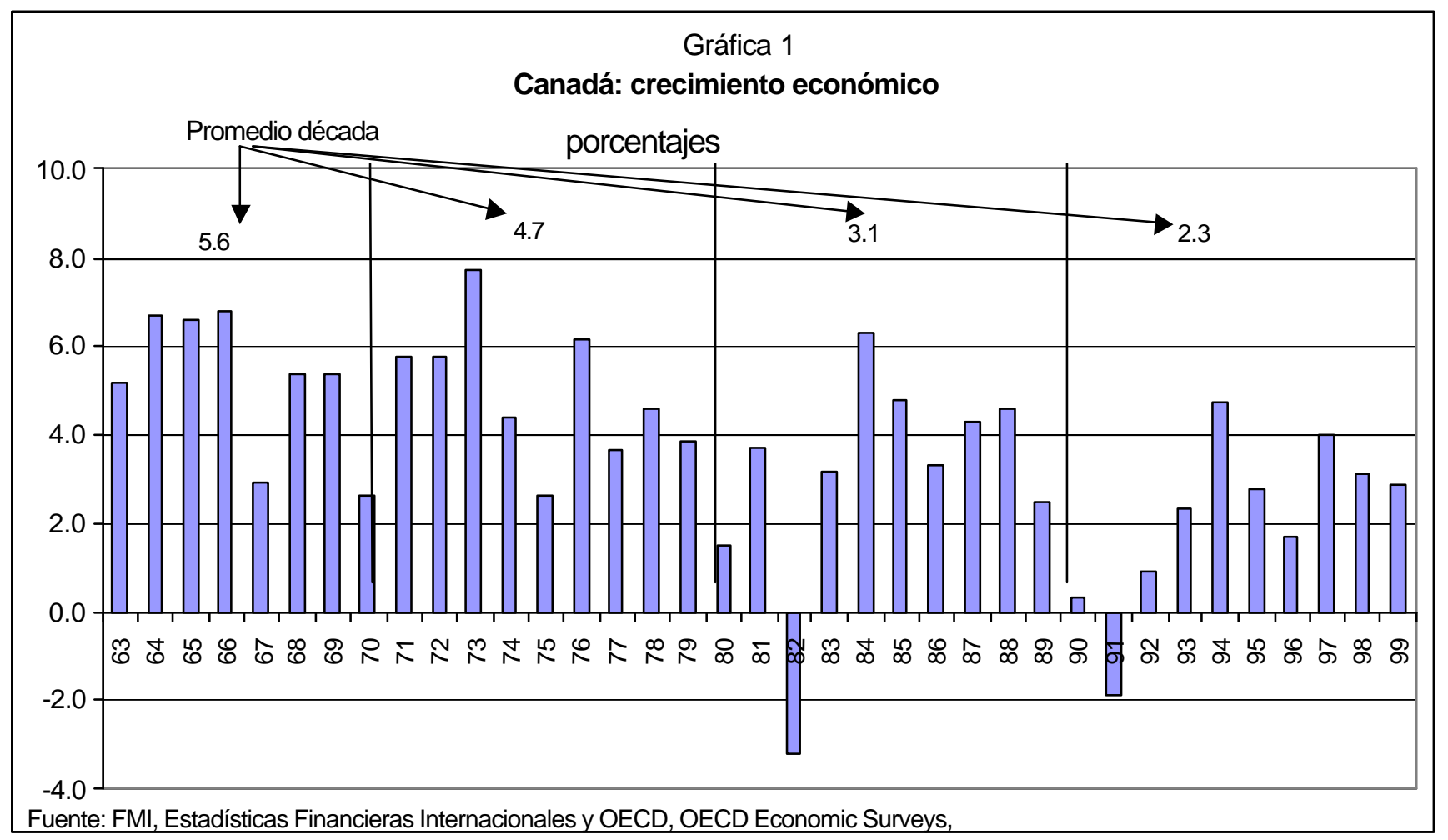


La región del Atlántico, con 2.4 millones de habitantes que constituyen 8 por ciento del total y de tamaño similar al de Francia, destaca actualmente por su infraestructura en telecomunicaciones y por la intensidad en el comercio electrónico. Nuevo Brunswick es la única provincia con frontera con Estados Unidos en la región. Tradicionalmente, la región ha exportado productos basados en recursos naturales y materiales procesados, aunque esta tendencia se ha revertido en los últimos años (cuadro 2).

A mediados de los noventa, de las cinco regiones, ésta es la que presentaba la menor participación en el PIB total, con una contribución de 6 por ciento, mientras empleaba a 7 por ciento de la fuerza laboral. El nivel de ingreso de sus habitantes, comparado a la media nacional, es el más bajo de las cinco regiones, representando alrededor de 73 por ciento del ingreso promedio nacional.

La región de Quebec es la segunda economía más importante de Canadá, después de Ontario, contribuyendo con 22 por ciento al PIB nacional y empleando a 24 por ciento de la fuerza de trabajo. En esta región habita una cuarta parte de la población del país, la cual posee un nivel de ingreso superior al de la región del Atlántico pero inferior al de la media nacional (cuadro 2).

Quebec es la única provincia donde el francés es la lengua oficial. El área del Gran Montreal concentra la mitad de la economía de la provincia y de la población. Los sectores líderes en la región de Quebec son el aero espacial, el de telecomunicaciones, el de farmaceúticos y el de biotecnología. Las empresas Bombardier y Nortel son líderes mundiales en las industrias aerospacial y en la de telecomunicaciones. De las exportaciones de Quebec, 83 por ciento se dirigen a Estados Unidos y la mayor parte de la inversión extranjera directa (IED) que recibe, proviene de ese país.

Continuando de este a oeste, Ontario es la región dominante del país tanto en términos de la población como por su influencia económica, política y cultural. Esta provincia alberga 40 por ciento de la población, que se distinguen por su diversidad étnica, ya que más de la mitad tienen origen diferente del francés o inglés. 40 por ciento del empleo a nivel nacional se concentra

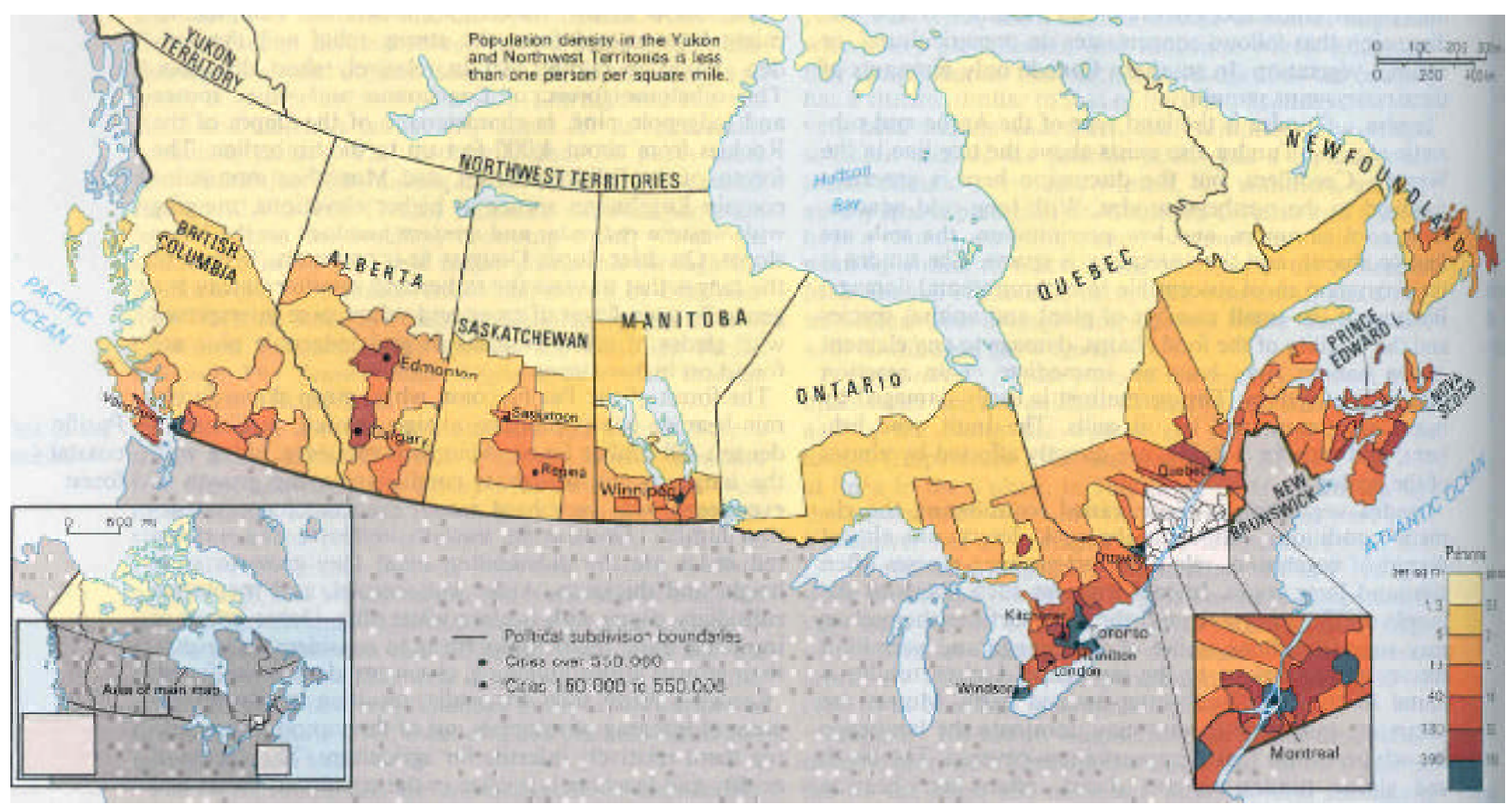

Fuente: The new enciclopaedia Britanica, vol. 15 1998, p. 448 
en esta zona y el ingreso de sus habitantes está por encima de la media nacional. El centro comercial del país es Toronto; en él se ubican la mitad de las instituciones financieras y la mayor parte de los bancos internacionales. La revista Fortune ha clasificado a Toronto como la mejor ciudad para los negocios.

La producción de Ontario constituye 40 por ciento del PIB nacional y 53 por ciento de la producción manufacturera. La economía está muy desarrollada y diversificada, ya que descansa tanto en la industria como en la agricultura y la minería. En la industria sobresalen los sectores de telecomunicaciones y el automotriz. En este último compite con Michigan como centro de ensamble automovilístico. Además, Ontario se distingue por las industrias del acero, de químicos, la aeroespacial, la alimenticia, la biotecnológica y la de la computación. Ontario es también el líder nacional en la producción agrícola de frutas, vegetales, granos, aves, lácteos y, recientemente, vinos, además de contribuir con 30 por ciento de la producción mineral y 20 por ciento de la forestal.

La fuerza laboral de Ontario ha sido considerada como la más educada y productiva del mundo. Alrededor de 90 por ciento de la mano de obra poseen nivel de bachillerato y la mitad de los egresados de ese nivel asisten a la universidad.

Las exportaciones de Ontario constituyen casi la mitad de su PIB, y al igual que el resto del país, está concentrado con Estados Unidos, siendo sus socios principales los estados de Michigan, Ohio, Indiana, Nueva York, Illinois, Tejas y California.

Siguiendo hacia el oeste se encuentra la región de las provincias, de las praderas y los territorios del Noroeste, ricas en recursos naturales. En esta zona se genera alrededor de una quinta parte de la producción nacional y en ella habitan 17 por ciento de la población del país. Sus pobladores gozan de un elevado nivel de ingreso, sobre todo los de la provincia de Alberta (cuadro 2). La zona concentra 80 por ciento de la tierra agrícola de Canadá y dos tercios de la producción mineral. En consecuencia, destacan la industria alimenticia, la del gas y la del petróleo. La región de las praderas exporta alrededor de 40 por ciento de su producción manufacturera y Estados Unidos es el destino de 80 por ciento de estas.

Finalmente, en la costa del Pacífico está la región de Columbia Británica y el Territorio Yukon. En ella habitan tres millones de personas y es la tercera más grande de Canadá. Los sectores que sobresalen en la región son el de la industria electrónica, la biotecnología y el turismo. Vancouver es el más grande y diversificado puerto de Canadá y la mayoría de las compañías asiáticas tienen agentes en Vancouver.

\section{Problemas actuales de la economía canadiense}

La economía canadiense inició la década de los noventa con un crecimiento casi nulo (en 1990) y se contrajo 2 por ciento el año siguiente. A partir de 1992 ha mostrado tasas superiores al 2 por ciento con excepción de 1996 (gráfica 1). Estas variaciones en el PIB arrojan un promedio de crecimiento de 2.3 por ciento en la década superior, en apenas 0.5 por ciento al de la población. El magro desempeño de la economía ha estado acompañado de elevadas tasas de desempleo que, en promedio, fueron de 9.4 por ciento entre 1994 y 1998. Con el objetivo de corregir el desempleo estructural que ha afectado a Canadá, el gobierno introdujo varias medidas entre las que destaca la modificación del seguro de desempleo (de 1971) por el Seguro de Empleo a partir de 1996, y según el cual se introducen requerimientos más estrictos de elegibilidad y se modifica la duración y monto de los beneficios del desempleo. ${ }^{3}$

Vinculado con lo anterior están las bajas tasas de crecimiento de la productividad laboral, la cual se ha visto influida por la adaptación lenta de nueva tecnología y el desfase entre el entrenamiento de la mano de obra y las nuevas capacidades requeridas por la industria. El retraso en el uso de robots y máquinas 
numéricamente controladas por computadora han causado rezagos en la calidad y los costos en los productos tradicionales y en los bienes con alto contenido en investigación. Por otra parte, la lenta adopción de nuevas tecnologías en el trabajo y en nuevos productos ha frenado la incursión canadiense en los mercados internacionales. ${ }^{4}$

Como señala Britton, el desarrollo económico de Canadá está concentrado en unas cuantas industrias importantes, mientras que las empresas que están concentradas espacialmente en Toronto y Montreal controlan una cuarta parte del mercado y de la producción. Así, 56 por ciento del stock de capital con que cuenta Canadá se localiza en Ontario y Quebec (Cuadro 2). La política económica seguida por las provincias (utilizando los subsidios, las compras gubernamentales y el control en los mercados de los consumidores) ha propiciado la división del mercado nacional y ha interferido con la movilidad de la mano de obra y de los productores.
Canadá tiene ante sí el reto de cerrar la brecha tecnológica y profundizar el proceso de industrialización, así como incorporar las regiones menos privilegiadas al desarrollo económico del país. La calidad de los recursos naturales, físicos y humanos con que cuenta lo sitúan en una posición de privilegio para superar el desafío.

\section{Notas}

1. US-Trade Data Bank, US Department of Commerce. Noviembre 1999. Varios artículos sobre Canadá: Background notes; World Factbook, etc.

2. Esta sección está basada principalmente en US-Trade Data Bank, US Department of Commerce, "Country Commercial Guide for Canada», Cap. IV, noviembre 1999, CD.

3. OECD, OECD Economic Surveys Canada 1999, pp. 5559.

4. Canada and the Global Economy. The Geography of Structural and Technological Change. Ed. Britton, John. Cap. 1, Introducción de Britton. McGill Queens University Press, 1996. 